\title{
Personalisiert, individualisiert, stratifiziert - Thoraxonkologie quo vadis?
}

\author{
Personalized, Individualized, Stratified - Thoracic Oncology quo vadis?
}

Autoren

Institut
M. Thomas, H. Bischoff, H. Grosch, M. Sianidou, M. Steins, M. Villalobos

Internistische Onkologie der Thoraxtumoren, Thoraxklinik am Universitätsklinikum Heidelberg

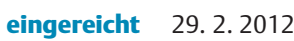
akzeptiert nach Revision 3. 3.2012

\section{Bibliografie}

Dol http://dx.doi.org/ 10.1055/s-0032-1308906 Online-Publikation: 24.4.2012 Pneumologie 2012; 66: 338-340 (c) Georg Thieme Verlag KG Stuttgart · New York ISSN 0934-8387

\section{Korrespondenzadresse} Prof. Dr. Michael Thomas Internistische Onkologie der Thoraxtumoren Thoraxklinik am Universitätsklinikum Heidelberg Amalienstraße 5 69126 Heidelberg michael.thomas@thoraxklinikheidelberg.de
Die Möglichkeiten zur „molekularen Diversifikation“ des nicht-kleinzelligen Lungenkarzinoms nehmen rasch zu. In Verbindung damit zeichnen sich spezifisch zuzuordnende Therapiemöglichkeiten durch Tyrosinkinaseinhibitoren oder Antikörper ab. In diesem Zusammenhang findet sich oft der Begriff der „personalisierten“ oder „individualisierten“ Krebstherapie. Dieser Kommentar versucht eine Standortbestimmung zu den Kontexten der „Personalisierung, Individualisierung und Stratifizierung“ in der Krebstherapie.

Die Therapiestrategien in der Onkologie sind in den letzten Jahren bei verschiedenen Tumorentitäten durch eine Zunahme der auf die molekularen Mechanismen der Erkrankung gerichteten Diagnose- und Behandlungsmöglichkeiten (Tyrosinkinaseinhibitoren, Antikörper) gekennzeichnet. Da die Interferenz mit einem molekularen Mechanismus sehr spezifisch sein kann und zudem der Einfluss molekularer Alterationen auf die Tumorpropagation, das Tumorwachstum oder Metastasierung in einer Tumorentität nicht vorhersagbar ist, sollten bereits in Phase-I/II-Studien Strategien zur Exploration einer prädiktiven Diagnostik verfolgt werden [1,2]. Gibt es aus präklinischen Analysen keinen Hinweis für einen „Link“ zwischen Substanz, möglichem Wirkmechanismus und Therapieeffekt, wäre dennoch zunächst eine weitere Exploration in präklinischen Modellen hilfreich. Die daraus abgeleiteten Hypothesen für eine mögliche prädiktive Diagnostik können dann in Phase-I/II-Studien begleitend validiert werden. Kann dies so nicht verfolgt werden, sollte der Akquise von Biomaterialien (Tumorgewebe, Blut) im Phase-I/II-Kontext höchste Priorität eingeräumt werden. So ist zumindest im Nachgang die Möglichkeit zur Exploration prädiktiver Parameter gegeben, um Hypothesen zur Charakterisierung von Patientengruppen mit bestmöglichem Therapieeffekt aufzustellen. Dies ist eine wichtige Voraussetzung, um so für Phase-III-Stu- dien Kriterien für den Patienteneinschluss oder doch zumindest die Patientenstratifikation festzulegen. Als Beispiele für diese Entwicklung können in der Krebstherapie die Behandlung der chronisch myeloischen Leukämie bzw. der gastrointestinalen Stromatumoren mit Imatinib, des kolorektalen Karzinoms mit Cetuximab sowie des Mammakarzinoms mit Trastuzumab angesehen werden.

\section{Stratifizierte Medizin}

$\nabla$

Durch prädiktive Diagnostik (Translokations-, Mutationsanalyse bzw. Nachweis einer Rezeptorüberexpression) können bei den vorgenannten Krankheitsbildern diejenigen Patienten charakterisiert werden, für die ein klinisch relevanter Effekt durch die jeweilige Therapie zu erwarten ist. Gerade beim metastasierten Lungenkarzinom ist in den nächsten Jahren auf dem Feld der „stratifizierten Medizin“ eine dynamische Entwicklung zu erwarten. Neben den aktivierenden Mutationen des EGF-Rezeptors [3,4] ist mit der EML4ALK-Translokation [5] bereits jetzt eine weitere molekulare Alteration beim pulmonalen Adenokarzinom beschrieben, die einer spezifischen Therapie zugänglich ist. Letztlich zeigt sich, dass bei entsprechender Analyse für 50-55\% der pulmonalen Adenokarzinome definierte molekulare Alterationen nachweisbar sind (KRAS, EGFR, BRAF, HER 2, PIK3CA, MET-Amplifikation, MEK1, N-RAS, AKT1, EML4-ALK) [6]. Auch ein Teil der pulmonalen Plattenepithelkarzinome lässt sich molekular definierten Gruppen zuordnen. Es zeichnet sich ab, dass in den nächsten Jahren Therapieansätze verfügbar werden, für die ein spezifischer Effekt durch Zuordnung zu molekularen Alterationen zu erwarten steht [7]. Neben dem klassischen Staging werden dann auch Punkte wie Biopsiegröße, strukturierter Aufbau der molekularpathologisch-diagnostischen Strategien 


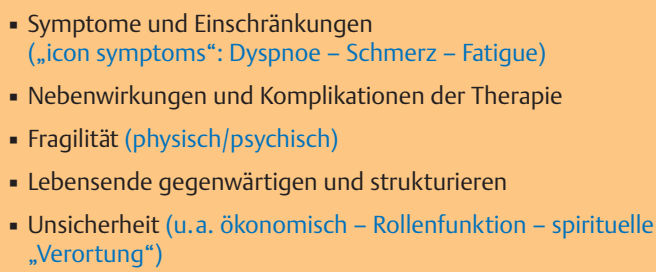

Abb. 1 Dimensionen der Belastung für Patienten/Angehörige beim metastasierten Lungenkarzinom.

2885 Tumorpatienten, davon 503 mit Lungenkarzinomen; retrospektive Analyse aus 2 Datenbanken (multizentrisch) mit Erhebung zur Lebensqualität (BIOQOL/Q-Score - Database)

\begin{tabular}{lllr}
\hline Gesamt & & & \\
\hline ECOG & $0 / 1$ & 2 & $3 / 4$ \\
\hline Behandler & $78 \%$ & $15 \%$ & $7 \%$ \\
\hline Patienten & $61 \%$ & $28 \%$ & $11 \%$ \\
\hline
\end{tabular}

\begin{tabular}{|lllr|}
\multicolumn{4}{|l}{ Lungenkarzinom } \\
\hline ECOG & $0 / 1$ & 2 & $3 / 4$ \\
\hline Behandler & $66 \%$ & $21 \%$ & $13 \%$ \\
\hline Patienten & $48 \%$ & $34 \%$ & $18 \%$ \\
\hline
\end{tabular}

Abb. 2 Einschätzung des Allgemeinzustandes gemäß ECOG-Klassifikation aus Patienten- und Behandlersicht. Adaptiert aus Lilienbaum et al. [10].

(Immunhistochemie, FISH-Analyse, PCR-Mutationsanalyse, Multiplexanalyse) sowie die indikative molekularpathologische Befundung bedeutsam werden [8].

Für die Konzepte der „stratifizierten Therapie“ wird häufig synonym der Begriff der „personalisierten oder individualisierten Krebstherapie" gebraucht. Der Ansatz zielt jedoch auf molekulare Alterationen im Tumor des Patienten und berührt damit die Person an sich nicht und auch nicht das, was ein Individuum ausmacht. Es handelt sich um die Behandlung von Patientengruppen, deren Tumoren definierte molekulare Merkmale tragen, also um ein stratifiziertes Therapiekonzept. Der Begriff der stratifizierten Medizin wird der Prägung dieses Paradigmas gerechter als die „personalisierte oder individualisierte Krebstherapie“.

„Personalisierte Medizin ist in Mode, weil sie einen Gegenpol zur naturwissenschaftlichen in Schemata denkenden modernen Hightech-Medizin zu sein vorgibt“, schreibt G. Maio in einer ethischen Betrachtung zu dieser Begrifflichkeit [9]. Er sieht die Gefahr, dass der „gegenwärtige Trend der Pharmakogenomik gerade nicht zur Aufwertung der Persönlichkeit, sondern im Gegenteil gerade zu deren Abwertung [führt]... Je mehr sachliche Informationen vorliegen, desto mehr wird man dem Irrtum verfallen, in der Erhebung dieser Sachlichkeit schon das Wesentliche über den Menschen ausgesagt zu haben ... Je mehr wir direkt in Zahlen ausdrücken können, desto mehr werden wir das nicht Zählbare, das nicht Abbildbare, das nicht Standardisierbare für irrelevant erachten. Man wird Zug um Zug denken, dass man der Individualität des Patienten dadurch gerecht wird, dass man seine individuellen Gene untersucht und man wird es immer mehr für verzichtbar halten, sich in ganz individueller Weise mit der Lebenswelt des Patienten auseinanderzusetzen.“

Gerade in der Thoraxonkologie ist es bedeutsam, dies jedoch im Blick zu behalten. Nahezu jeder zweite Patient mit einem Lungenkarzinom weist zum Diagnosezeitpunkt bereits eine metastasierte Erkrankung auf - die Hälfte dieser Patienten lebt mit den derzeit verfügbaren Therapiemöglichkeiten nicht länger als zehn bis zwölf Monate. Diese Perspektive ist mit erheblichen Belastungen für Patient und Familie verbunden ( $\bullet$ Abb.1). Dazu kommt, dass allein die kumulative Inzidenz von Hirnmetastasen sowie Knochenmetastasen über diesen Zeitraum hinweg bei $40-50 \%$ liegt. Durch die damit verbundene Symptomatik wird für viele Patienten und deren Familien in dieser kurzen Zeit physische Fragilität, eine Veränderung der Persönlichkeit und eingeschränkte Mobilität oder Immobilität rasch erfahrbar.

\section{Personalisierte Medizin}

Um medizinische Maßnahmen an patientenseitige Faktoren und Einschränkungen anzupassen, bedarf es einer entsprechenden Wahrnehmung und Einschätzung. Bereits bei der Einschätzung des Allgemeinzustandes jedoch kann es erhebliche Differenzen zwischen Patienten- und Behandlersicht geben [10] ( $\bullet$ Abb.2). Die Möglichkeit der nicht hinlänglichen Konkordanz sollte im Bewusstsein bleiben und im klinischen Alltag der Patient selbst immer auch nach seiner Einschätzung befragt werden. Darüber hinaus ist die Kenntnis und Bewertung von Komorbiditäten, von physischen Einschränkungen, der sozialen Situation und unter Umständen auch die Einschätzung der mentalen Leistungsfähigkeit für die Therapiewahl und Anpassung der Behandlung bedeutsam. Letztlich kann so auch eine Einschätzung zu erwartender Toxizitäten im Nachgang zu einer Chemotherapie - und demgemäß eine Anpassung der Therapieintensität - möglich werden [11]. Zudem kann das Komorbiditätsausmaß für die Prognose von größerer Bedeutung sein als die Krebserkrankung selbst. In solchen Konstellationen stellt sich die Frage nach der demgemäß bestmöglich angepassten tumorspezifischen Therapie $[12,13]$. So gesehen kann mit dem Begriff der „personalisierten Krebstherapie“ wohl am besten die Berücksichtigung patientenseitiger Faktoren, von Komorbiditäten, von alters- oder erkrankungsbedingten Einschränkungen der Organfunktion wie auch des sozialen Umfeldes bei der Therapiewahl und -planung reflektiert werden $(\odot$ Abb. 3). All diese Faktoren wiederum wirken auf Ausmaß und Intensität der durch die Therapie zu erwartenden Nebenwirkungen und Komplikationen zurück und sind für eine auf die betroffene Person gut „zugeschnittene“ Behandlung wichtig. Insbesondere in der palliativen Therapiesituation ist in diesem Prozess die

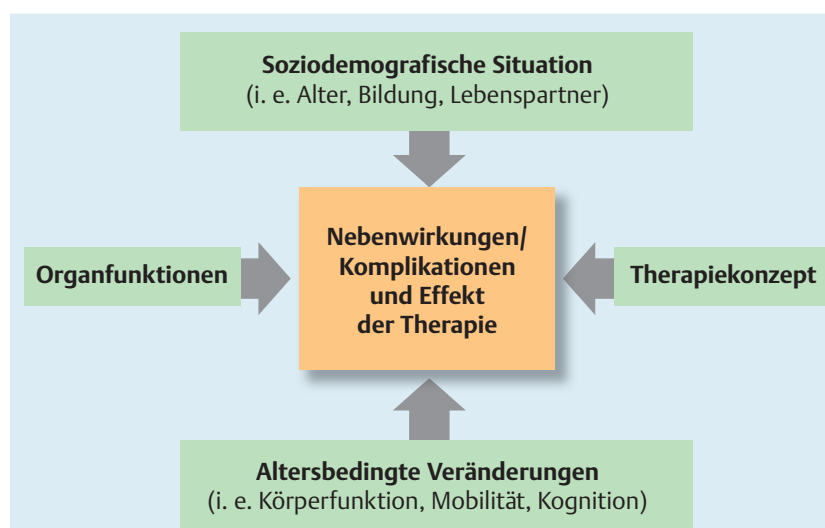

Abb. 3 „Personalisierung“ der onkologischen Therapie: Integration verschiedener Einflussgrößen unter Berücksichtigung der Patientenpräferenz für die Einschätzung von Effekt sowie Komplikationen und Nebenwirkungen einer Therapiemaßnahme. 
- Behandlungsplan konsistent zu Evidenz (Guidelines) u. Präferenz (informierter Patient)

- Fortlaufende Gewichtung (Evidenz-Präferenz) im Krankheitsverlauf

- Offener Dialog von Anfang an (Behandler - Patient/Angehörige)

- Prognose

- Effekt/Nebenwirkungen von (Therapie) Maßnahmen

- Effekt/Nebenwirkungen von Alternativen

- Behandlungsziele in der Terminalphase

Abb. 4 Thesen der ASCO (American Society of Clinical Oncology) zur individualisierten Betreuung bei fortgeschrittener Krebserkrankung Prinzipien. Adaptiert aus Peppercorn et al. [14].

- Skizze eines „worst case/best case“-Szenarios

$\Rightarrow$ Verständnis Verlauf/Prognose

- Bewertung und Erläuterung möglicher Therapieoptionen (Nutzen/Nebenwirkungen)

- Therapiemaßnahmen sollten (angesichts ihrer Limitation) als Option und nicht als Notwendigkeit gesehen werden

- Palliative Konzepte (auch ohne „cancer-directed treatment“) sollten gleichermaßen unter Berücksichtigung von Nutzen/ Nebenwirkungen erläutert und bewertet werden

- Fortlaufender Diskurs Zu

- Prognose

- aktuellem Befinden

- Lebensqualität

- Therapiezielen

Abb. 5 Thesen der ASCO (American Society of Clinical Oncology) zur individualisierten Betreuung bei fortgeschrittener Krebserkrankung Umsetzung. Adaptiert aus Peppercorn et al. [14]

Kenntnis der patientenseitigen Präferenz und Haltung gleichermaßen bedeutsam wie die Berücksichtigung der vorgenannten Faktoren und Einflussgrößen.

\section{Individualisierte Medizin}

Das Konzept der individualisierten Betreuung gerade für Patienten in der metastasierten Tumorsituation und mit einer kurzen Überlebenserwartung ist in einem Thesenpapier der American Society of Clinical Oncology (ASCO) gut dargestellt [14]. Diese Patienten müssen mit komplexen Herausforderungen in physischer, psychologischer, sozialer und spiritueller Dimension umgehen. Individualisierte Betreuung bedeutet, die für den einzelnen Patienten notwendigen Maßnahmen, die individuellen Ziele und die individuellen Präferenzen während des gesamten Krankheitsverlaufes auf den genannten Ebenen jeweils adäquat zu erfassen und darauf einzugehen ( $\bullet$ Abb.4). Neben krankheitsspezifischen Therapiemaßnahmen, symptomorientierter Behandlung und Maßnahmen zum Erhalt der Lebensqualität ist für den Krankheitsverlauf im gleichen Maß eine realitätsbezogene, stützende Kommunikationslinie von Bedeutung. Die Autoren mahnen an, dass dies oft nicht der Fall ist und eine realitätsbezogene Gesprächsführung zur Prognose der Erkrankung, zum potenziel- len Nutzen krankheitsspezifischer Maßnahmen, zur potenziellen Bedeutung palliativmedizinischer Maßnahmen mit oder ohne Verbindung zu alternativen Behandlungskonzepten oft nur unzureichend erfolgt ( $\bullet$ Abb.5). Gerade beim metastasierten Lungenkarzinom konnte in einer randomisierten Studie gezeigt werden, dass regelmäßige Gesprächskontakte mit Fokus auf a) Information zu und Verständnis der Erkrankung - b) Behandlung von Symptomen - c) Vermittlung von Strategien zum Umgang mit der Situation an den Patienten und seine Angehörigen - d) Festlegung der zu erfolgenden Maßnahmen am Lebensende - zu einer signifikanten Verbesserung der Lebensqualität, Reduktion der psychischen Belastung in Form einer Depression sowie situations- und patientengemäßen Managementstrategien am Lebensende führen. Darüber hinaus konnte die mediane Überlebenszeit signifikant verbessert werden [15].

\section{Interessenkonflikt}

Ein Interessenkonflikt der Autoren im Hinblick auf den publizierten Standpunkt liegt nicht vor.

\section{Literatur}

1 Lara PN Jr, Douillard JY, Nakagawa K. Randomized phase III placebocontrolled trial of carboplatin and paclitaxel with or without the vascular disrupting agent vadimezan (ASA404) in advanced non-smallcell lung cancer. J Clin Oncol 2011; 29: 2965-2971

2 Lorusso PM, Boerner SA, Hunsberger S. Clinical development of vascular disrupting agents: what lessons can we learn from ASA404? J Clin Oncol 2011; 29: $2952-2955$

3 Mok TS, Wu YL, Thongprasert $S$ et al. Gefitinib or carboplatin-paclitaxel in pulmonary adenocarcinoma. N Engl J Med 2009; 361: 947-957

4 Rosell $R$, Gervais $R$, Vergnenegre $A$ et al. Erlotinib versus chemotherapy (CT) in advanced non-small cell lung cancer (NSCLC) patients (p) with epidermal growth factor receptor (EGFR) mutations: Interim results of the European Erlotinib Versus Chemotherapy (EURTAC) phase III randomized trial. J Clin Oncol 2011; 29: 476 (suppl., abstr. 7503)

5 Kwak EL, Bang YJ, Camidge DR et al. Anaplastic lymphoma kinase inhibition in non-small-cell lung cancer. N Engl J Med 2010; 363: $1693-1703$

6 Kris MG, Johnson BE, Kwiatkowski DJ et al. Identification of driver mutations in tumor specimens from 1,000 patients with lung adenocarcinoma: The NCl's Lung Cancer Mutation Consortium (LCMC). J Clin Oncol 2011; 29: 477 (suppl., abstr. 7506)

7 Pao W, Girard N. New driver mutations in non-small-cell lung cancer. Lancet Oncol 2011; 12: 175-180

8 Muley T, Herth FJ, Schnabel $P$ et al. From tissue to molecular phenotyping: Pre-analytical requirements Heidelberg Experience. Transl Lung Cancer Res 2011; 26: DOI 10.3978/j.issn.2218-6751-2011.12.07

9 Maio G. Chancen und Grenzen der personalisierten Medizin - eine ethische Betrachtung. GGW 2012; 12: 15-19

10 Lilienbaum RC, Cashy J, Hensing TA et al. Prevalence of poor performance status in lung cancer patients: implications for research. J Thorac Oncol 2008; 3: 125-129

11 Hurria A, Togawa K, Mohile SG et al. Predicting chemotherapy toxicity in older adults with cancer: a prospective multicenter study. J Clin Oncol 2011; 29: 3457-3465

12 Ring A, Sestak I, Baum M et al. Influence of comorbidities and age on risk of death without recurrence: a retrospective analysis of the Arimidex, Tamoxifen Alone or in Combination trial. J Clin Oncol 2011; 29: $4266-4272$

13 Hurria A. Embracing the complexity of comorbidity. J Clin Oncol 2011; 29: 4217-4218

14 Peppercorn JM, Smith TJ, Helft PR et al. American society of clinical oncology statement: toward individualized care for patients with advanced cancer. J Clin Oncol 2011; 29: 755-760

15 Temel JS, Greer JA, Muzikansky A et al. Early palliative care for patients with metastatic non-small-cell lung cancer. N Engl J Med 2010; 363: $733-743$ 